\title{
Proton-hole and core-excited states in the semi-magic nucleus ${ }^{131} \ln _{82}$
}

J. Taprogge $\mathrm{e}^{1,2,3}$, A. Jungclaus ${ }^{1, a}$, H. Grawe ${ }^{4}$, I.N. Borzov ${ }^{5,6}$, S. Nishimura ${ }^{3}$, P. Doornenbal ${ }^{3}$, G. Lorusso $^{3,7,8}$ G.S. Simpson ${ }^{9}$, P.-A. Söderström ${ }^{3}$, T. Sumikama ${ }^{10}$, Z.Y. Xu ${ }^{11}$, H. Baba ${ }^{3}$, F. Browne ${ }^{12,3}$, N. Fukuda $^{3}$, R. Gernhäuser $^{13}$, G. Gey ${ }^{9,14,3}$, N. Inabe ${ }^{3}$, T. Isobe ${ }^{3}$, H.S. Jung ${ }^{15, b}$, D. Kameda ${ }^{3}$, G.D. Kim ${ }^{16}$, Y.-K. Kim ${ }^{16,17}$, I. Kojouharov ${ }^{6}$, T. Kubo ${ }^{3}$, N. Kurz ${ }^{6}$, Y.K. Kwon ${ }^{16}$, Z. Li ${ }^{18}$, H. Sakurai ${ }^{3,11}$, H. Schaffner ${ }^{6}$, Y. Shimizu ${ }^{3}$, K. Steiger ${ }^{13}$, H. Suzuki ${ }^{3}$, H. Takeda ${ }^{3}$, Zs. Vajta ${ }^{19,3}$, H. Watanabe ${ }^{3}$, J. Wu ${ }^{18,3}$, A. Yagi ${ }^{20}$, K. Yoshinaga ${ }^{21}$, G. Benzoni ${ }^{22}$, S. Bönig $^{23}$, K.Y. Chae ${ }^{24}$, L. Coraggio ${ }^{25}$, J.-M. Daugas ${ }^{26}$, F. Drouet ${ }^{9}$, A. Gadea ${ }^{27}$, A. Gargano ${ }^{25}$, S. Ilieva ${ }^{23}$, N. Itaco $^{28}$, F.G. Kondev ${ }^{29}$, T. Kröll ${ }^{23}$, G.J. Lane ${ }^{30}$, A. Montaner-Pizá ${ }^{27}$, K. Moschner ${ }^{31}$, D. Mücher ${ }^{13}$, F. Naqvi ${ }^{32}$, M. Niikura ${ }^{11}$,

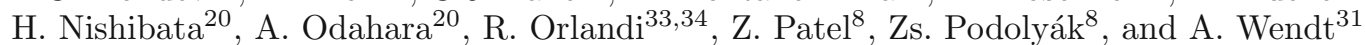

1 Instituto de Estructura de la Materia, CSIC, E-28006 Madrid, Spain

2 Departamento de Física Teórica, Universidad Autónoma de Madrid, E-28049 Madrid, Spain

3 RIKEN Nishina Center, RIKEN, 2-1 Hirosawa, Wako-shi, Saitama 351-0198, Japan

4 GSI Helmholtzzentrum für Schwerionenforschung GmbH, 64291 Darmstadt, Germany

5 Kurchatov Institute, 123182 Moscow, Russia

6 Joint Institute for Nuclear Research, 141980 Dubna, Russia

7 National Physical Laboratory, NPL Teddington, Middlesex TW11 0LW, UK

8 Department of Physics, University of Surrey, Guildford GU2 7XH, UK

9 LPSC, Université Joseph Fourier Grenoble 1, CNRS/IN2P3, Institut National Polytechnique de Grenoble, F-38026 Grenoble Cedex, France

10 Department of Physics, Tohoku University, Aoba, Sendai, Miyagi 980-8578, Japan

11 Department of Physics, University of Tokyo, Hongo 7-3-1, Bunkyo-ku, 113-0033 Tokyo, Japan

12 School of Computing, Engineering and Mathematics, University of Brighton, Brighton BN2 4GJ, UK

13 Physik Department E12, Technische Universität München, D-85748 Garching, Germany

14 Institut Laue-Langevin, B.P. 156, F-38042 Grenoble Cedex 9, France

15 Department of Physics, Chung-Ang University, Seoul 156-756, Korea

16 Rare Isotope Science Project, Institute for Basic Science, Daejeon 305-811, Korea

17 Department of Nuclear Engineering, Hanyang University, Seoul 133-791, Korea

18 School of Physics and State key Laboratory of Nuclear Physics and Technology, Peking University, Beijing 100871, China

19 MTA Atomki, P.O. Box 51, Debrecen H-4001, Hungary

20 Department of Physics, Osaka University, Machikaneyama-machi 1-1, Osaka 560-0043 Toyonaka, Japan

21 Department of Physics, Faculty of Science and Technology, Tokyo University of Science, 2641 Yamazaki, Noda, Chiba, Japan

22 INFN, Sezione di Milano, via Celoria 16, I-20133 Milano, Italy

23 Institut für Kernphysik, Technische Universität Darmstadt, D-64289 Darmstadt, Germany

24 Department of Physics, Sungkyunkwan University, Suwon 440-746, Korea

25 Istituto Nazionale di Fisica Nucleare, Complesso Universitario di Monte S. Angelo, I-80126 Napoli, Italy

26 CEA, DAM, DIF, 91297 Arpajon cedex, France

27 Instituto de Física Corpuscular, CSIC-Univ. of Valencia, E-46980 Paterna, Spain

28 Seconda Università di Napoli, Dipartimento di Matematica e Fisica, 2-81100 Caserta, Italy

29 Nuclear Engineering Division, Argonne National Laboratory, Argonne, IL 60439, USA

30 Department of Nuclear Physics, Research School of Physical Sciences and Engineering, Australian National University, Canberra, A.C.T. 0200, Australia

31 IKP, University of Cologne, D-50937 Cologne, Germany

32 Wright Nuclear Structure Laboratory, Yale University, New Haven, CT 06520-8120, USA

33 Instituut voor Kern- en StralingsFysica, K.U. Leuven, B-3001 Heverlee, Belgium

34 Advanced Science Research Center, Japan Atomic Energy Agency, Tokai, Ibaraki, 319-1195, Japan 


\begin{abstract}
The $\beta$ decay of the $N=83$ nucleus ${ }^{131} \mathrm{Cd}$ has been studied at the RIBF facility at the RIKEN Nishina Center. The main purpose of the study was to identify the position of the $1 p_{3 / 2}$ and $0 f_{5 / 2}$ protonhole states and the energies of core-excited configurations in the semi-magic nucleus ${ }^{131}$ In. From the radiation emitted following the $\beta$ decay, a level scheme of ${ }^{131}$ In was established and the $\beta$ feeding to each excited state determined. Similarities between the single-particle transitions observed in the $\beta$ decays of the $N=83$ isotones ${ }^{132} \mathrm{In}$ and ${ }^{131} \mathrm{Cd}$ are discussed. Finally the excitation energies of several core-excited configurations in ${ }^{131} \mathrm{In}$ are compared to QRPA and shell-model calculations.
\end{abstract}

\section{Introduction}

The properties of nuclei at a double closure of both proton and neutron shells are of fundamental importance for the understanding of nuclear structure. The one-valenceparticle nuclei, possessing or lacking only one nucleon in otherwise empty or filled shells, give the energies of singleparticle (SPE) or single-hole (SHE) states which are important empirical parameters in any microscopic description of nuclear properties of atomic nuclei. Nuclei with two particles outside a doubly-magic core provide direct information on the correlations between pairs of nucleons occupying orbitals close to the Fermi surface, namely on the two-body effective interaction. Finally, doubly-closed nuclei themselves and one-valence-particle nuclei may give a direct measure of the size of the shell gaps as well as information about additional two-body matrix elements of the effective interaction through the study of core-excited two- and three-quasiparticle states, respectively, in which one nucleon is excited across either the neutron or the proton shell gap.

In the ${ }^{132} \mathrm{Sn}$ region, comprehensive data exist for the neutron and proton single-particle states in ${ }^{133} \mathrm{Sn}$ and ${ }^{133} \mathrm{Sb}$ as well as for the neutron-hole states in ${ }^{131} \mathrm{Sn}[1-3]$. With respect to the proton-hole states, experimental information was limited until recently to the $0 g_{9 / 2}$ and $1 p_{1 / 2}$ states $[4,5]$. Core-excited states at high excitation energies have been identified in the valence nuclei ${ }^{131} \mathrm{Sn},{ }^{133} \mathrm{Sb}$, and ${ }^{131}$ In $[2,3,6,7]$ while this information is still lacking for ${ }^{133} \mathrm{Sn}$.

We have investigated the $\beta$ decay of ${ }^{131} \mathrm{Cd}$ with the main purpose to determine the position of the still missing $1 p_{3 / 2}$ and $0 f_{5 / 2}$ proton-hole states and to observe excited states belonging to three-quasiparticle configurations in ${ }^{131} \mathrm{In}$. This decay is expected to be dominated by the allowed $\nu 0 g_{7 / 2} \rightarrow \pi 0 g_{9 / 2}$ Gamow-Teller (GT) transition (see fig. 1) which is observed in the decay of many other Cd and In isotopes with $N \leq 82$ [8-11]. However, as already observed in the case of ${ }^{132}$ In [12], the only $\beta$ decay of a $Z<50, N>82$ nucleus experimentally studied so far, also the first-forbidden (ff) $\nu 0 h_{11 / 2} \rightarrow \pi 0 g_{9 / 2}$ singleparticle transition should play a major role. Furthermore, the decay of ${ }^{131} \mathrm{Cd}_{83}$ constitutes a unique case to study the contribution of the high-energy ff transition which transforms a neutron occupying the $1 f_{7 / 2}$ orbital above the $N=82$ shell closure into a proton in the $0 g_{9 / 2}$ orbital below the $Z=50$ shell gap. Note that the ff transitions from the $\left(2 s_{1 / 2}, 1 d_{3 / 2}\right)$ neutron to the $\left(1 p_{1 / 2}, 1 p_{3 / 2}\right)$ proton orbitals (omitted in fig. 1) are of minor importance and have not been observed in the neighboring nuclei.

First results of the present experiment, concerning the energy of the $1 p_{3 / 2}$ single-proton state, have already been presented in ref. [13]. With the present paper, we shall provide a full account of the experimental information. The obtained results are then discussed within the framework of two different theoretical approaches, namely the quasiparticle random-phase approximation and the nuclear shell model.

\section{Experiment}

Radioactive ${ }^{131} \mathrm{Cd}$ ions were produced via fission of a ${ }^{238} \mathrm{U}$ beam with a kinetic energy of $345 \mathrm{MeV} / u$ and an average intensity of $8-10 \mathrm{pnA}$ on a Be target at the Radioactive Isotope Beam Factory (RIBF) at RIKEN in the framework of the EURICA project $[14-16]$. The produced ${ }^{131} \mathrm{Cd}$ ions were identified in-flight event by event by the BigRIPS separator [17] on the basis of the measured energy loss, $\Delta E$, time of flight, TOF, and magnetic rigidity, $B \rho$. Details about the identification procedure can be found in ref. [18]. Two different settings of BigRIPS were used, one optimized for the maximum transmission of ${ }^{136} \mathrm{Sn}$ and the other one tuned to predominantly transmit ${ }^{128} \mathrm{Pd}$. For a particle identification plot from this experiment we refer the reader to fig. 2 of our recent article reporting on the decay of ${ }^{133} \mathrm{Cd}[19]$. In total about $1.5 \times 10^{5}{ }^{131} \mathrm{Cd}$ ions were identified (about $9.2 \times 10^{4}$ in the ${ }^{136} \mathrm{Sn}$ and $5.7 \times 10^{4}$ in the ${ }^{128} \mathrm{Pd}$ setting), transported through the ZeroDegree spectrometer (ZDS) and finally implanted into the WAS3ABi (Wide-range Active Silicon Strip Stopper Array for $\beta$ and ion detection) array positioned at the focal plane of the ZDS. The WAS3ABi detector [14-16] consists of eight closely packed double-sided silicon strip detectors (DSSSD) with an area of $60 \times 40 \mathrm{~mm}^{2}$, a thickness of $1 \mathrm{~mm}$ and a segmentation of 40 horizontal and 60 vertical strips each. The resulting $1 \times 1 \mathrm{~mm}^{2}$ pixelation allows for a spatial correlation between the implanted ion and the detection of $\beta$-decay electrons. All decay events detected in WAS3ABi were stored and correlated off-line in space and time with the implanted ions. The $\gamma$ rays emitted following the $\beta$ decay of the radioactive nuclei were detected by the EURICA array, which comprises 84 germanium crystals

\footnotetext{
a e-mail: andrea.jungclaus@csic.es

b Present address: Department of Physics, University of
} Notre Dame, Notre Dame, IN 46556, USA. 


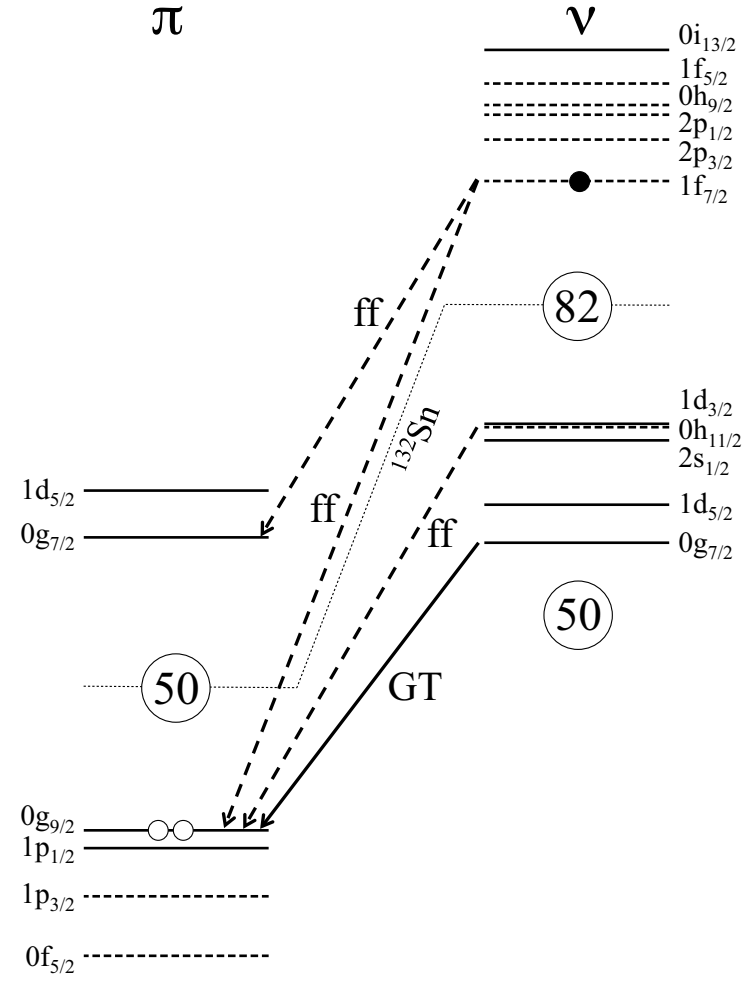

Fig. 1. Schematic view of the single-particle orbitals which are relevant in the ${ }^{132} \mathrm{Sn}$ region. The ground state configuration of ${ }^{131} \mathrm{Cd}$ is shown with open (proton holes) and filled (neutron particle) dots. The GT and ff single-particle decays discussed in the present work are indicated by solid and dashed arrows, respectively.

(12 large-volume Cluster detectors [20] from the former EUROBALL spectrometer [21]) arranged in a close geometry around the Si detectors. Energy and efficiency calibrations have been performed using ${ }^{133} \mathrm{Ba}$ and ${ }^{152} \mathrm{Eu}$ calibration sources and $\gamma$ rays from ${ }^{132} \mathrm{Sn}$ emitted following the $\beta$ decay of ${ }^{132}$ In for which absolute intensities are known from ref. [12]. For $\gamma$-ray energies above $4 \mathrm{MeV}$ extrapolations have to be relied upon. Systematic efficiency uncertainties of $5 \%$ for $E_{\gamma}<1.5 \mathrm{MeV}, 10 \%$ for $E_{\gamma}=1.5-4.0 \mathrm{MeV}$ and $20 \%$ for $E_{\gamma}>4.0 \mathrm{MeV}$ as well as energy uncertainties of $0.2 \mathrm{keV}$ for $E_{\gamma}<1.0 \mathrm{MeV}, 0.6 \mathrm{keV}$ for $E_{\gamma}=1.0-3.5 \mathrm{MeV}$ and $1.0 \mathrm{keV}$ for $E_{\gamma}>3.5 \mathrm{MeV}$ have been taken into account. After applying appropriate add-back algorithms (summing the energies of neighboring crystals) the photopeak efficiency of the EURICA array amounts to $8.5(4) \% / 2.9(3) \%$ for a $\gamma$-ray energy of $1 \mathrm{MeV} / 4 \mathrm{MeV}$.

\section{Data analysis and results}

Figure 2 shows the $\gamma$-ray spectrum observed in prompt coincidence with the first decay event after the implantation of ${ }^{131} \mathrm{Cd}$ ion in WAS3ABi. The time window between the implantation and the decay was limited to $300 \mathrm{~ms}$ corresponding to three times the half-life of ${ }^{131} \mathrm{Cd}$,
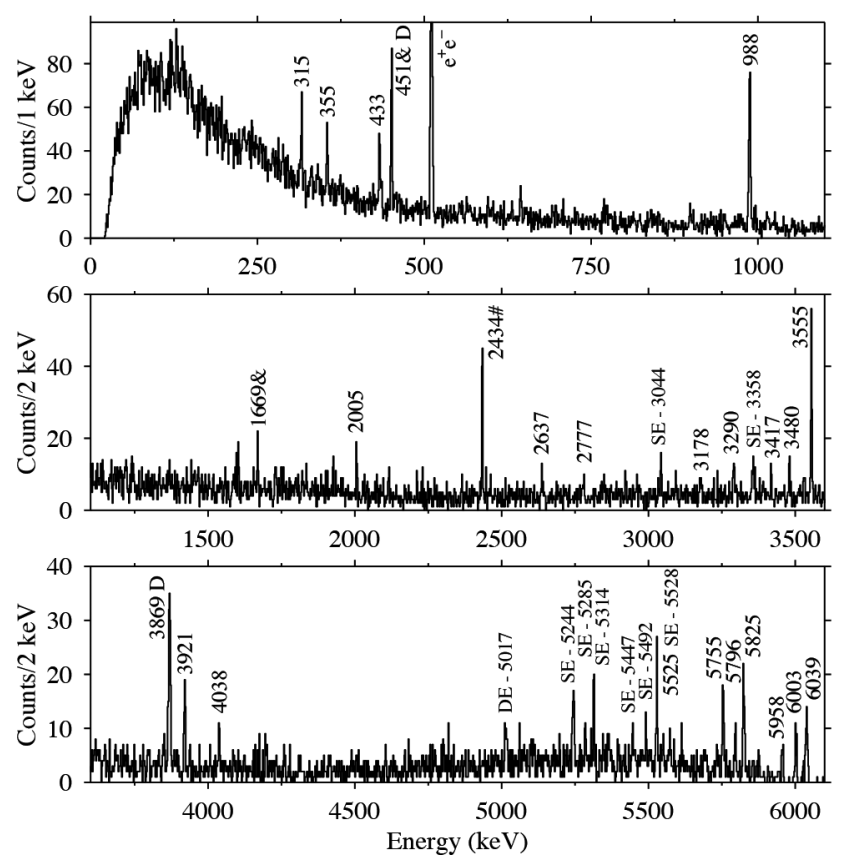

Fig. 2. A $\gamma$-ray spectrum recorded in coincidence with the first $\beta$ decay observed in WAS3ABi within $300 \mathrm{~ms}$ after the implantation of a ${ }^{131} \mathrm{Cd}$ ion and requiring that the implantation and the decay occurred in the same DSSSD and with a maximum position difference of $1 \mathrm{~mm}$ in the horizontal and vertical directions. The most intense transitions are labelled by their energies. The 451 and $1669 \mathrm{keV}$ lines (labelled \&) correspond to known transitions in ${ }^{130} \mathrm{In}$, populated either after the $\beta$-delayed neutron emission of ${ }^{131} \mathrm{Cd}$ or due to reactions occurring in beam detectors, degraders or the implantation detector after the ion identification, while the $2434 \mathrm{keV}$ transition (labelled \#) originates from the ${ }^{131} \mathrm{In} \rightarrow{ }^{131} \mathrm{Sn}$ daughter decay. The labels "SE" and "DE" indicate single- and double-escape lines, respectively, and "D" identifies doublet structures.

$T_{1 / 2}=98(2) \mathrm{ms}[22]$ (note that this value was accidentally quoted as $98.0(2) \mathrm{ms}$ in [22]). Only those events were considered in which the implantation and the decay were detected in the same $\mathrm{Si}$ detector and the distance between them in the two directions perpendicular to the beam axis was smaller or equal to $1 \mathrm{~mm}$. Under these conditions the efficiency for the detection of the $\beta$ decay amounts to about $33 \%$. Note that the requirements for the correlation in space and time between the ion implantation and the decay are slightly different from the ones applied to produce the spectrum shown in fig. 2 of ref. [13]. The most intense line in the spectrum is the one at $988 \mathrm{keV}$. As discussed in detail in ref. [13] this line is also observed in the decay of ${ }^{132} \mathrm{Cd}$ and was assigned as the transition from the $1 p_{3 / 2}$ proton single-particle state, at an excitation energy of $1353 \mathrm{keV}$, to the $1 p_{1 / 2} \beta$-decaying level at $365 \mathrm{keV}$ in ${ }^{131} \mathrm{In}$. In addition, two groups of intense lines are observed in the energy ranges $3.5-4.1$ and $5.5-6.1 \mathrm{MeV}$, respectively.

Finally, a number of additional weaker transitions are visible in fig. 2 , too. The energies and relative intensities of all $\gamma$ rays which have been assigned to the $\beta$ decay of 
Table 1. Energies, relative intensities and absolute intensities per parent decay of $\gamma$ rays observed in the $\beta$ decay of ${ }^{131} \mathrm{Cd}$. In the columns 4 and 5 the tentative placement of the transitions in the level scheme of ${ }^{131} \mathrm{In}$ is indicated.

\begin{tabular}{|c|c|c|c|c|}
\hline$E_{\gamma}(\mathrm{keV})$ & $I_{\gamma}(\%)$ & $\%$ per decay & $E_{i}(\mathrm{keV})$ & $E_{f}(\mathrm{keV})$ \\
\hline $315.0(2)$ & $25(12)$ & $2.2(10)$ & $3869(1)$ & $3555(1)$ \\
\hline $355.0(2)^{a}$ & $12(4)$ & $1.1(4)$ & - & - \\
\hline $433.2(2)$ & $27(7)$ & $2.4(6)$ & $5958(1)$ & $5525(1)$ \\
\hline $451(2)^{a, b}$ & $11(5)$ & $1.0(5)$ & - & - \\
\hline $987.9(2)^{*}$ & $100(15)$ & $8.8(17)$ & $1353(8)$ & $365(8)$ \\
\hline $2004.7(7)^{a}$ & $18(5)$ & $1.6(5)$ & - & - \\
\hline $2636.7(7)$ & $7(3)$ & $0.6(2)$ & $3990(8)$ & $1353(8)$ \\
\hline $2776.8(7)$ & $12(3)$ & $1.0(5)$ & $4130(8)$ & 1353(8) \\
\hline $3177.6(13)$ & $15(5)$ & $1.3(4)$ & $4531(8)$ & $1353(8)$ \\
\hline $3290.1(7)$ & $26(8)$ & $2.3(7)$ & $4644(8)$ & $1353(8)$ \\
\hline $3417.0(7)$ & $8(4)$ & $0.7(4)$ & $4770(8)$ & $1353(8)$ \\
\hline $3480.0(7)$ & $33(8)$ & $2.9(7)$ & $3480(1)$ & 0 \\
\hline $3555.2(10)^{*}$ & $98(16)$ & $8.6(15)$ & $3555(1)$ & 0 \\
\hline $3869(3)^{b, *}$ & $115(19)$ & $10.1(16)$ & $3869(3)^{c}$ & 0 \\
\hline $3920.6(11)$ & $46(12)$ & $4.1(10)$ & 3921(1) & 0 \\
\hline $4038.0(10)$ & $36(15)$ & $3.2(13)$ & $4038(1)$ & 0 \\
\hline $5525.0(11)$ & $29(9)$ & $2.5(8)$ & $5525(1)$ & 0 \\
\hline $5754.7(11)$ & $64(18)$ & $5.6(16)$ & $5755(1)$ & 0 \\
\hline $5796.0(11)$ & $29(9)$ & $2.5(8)$ & $5796(1)$ & 0 \\
\hline $5824.7(10)$ & $87(24)$ & $7.7(21)$ & $5825(1)$ & 0 \\
\hline $5958.3(12)$ & $25(9)$ & $2.2(7)$ & $5958(1)$ & 0 \\
\hline $6002.8(11)$ & $33(13)$ & $2.9(12)$ & $6003(1)$ & 0 \\
\hline $6039.2(10)^{*}$ & $63(20)$ & $5.5(18)$ & $6039(1)$ & 0 \\
\hline
\end{tabular}

${ }^{a}$ Not placed in the level scheme shown in fig. 5 .

${ }^{b}$ Doublet structure

${ }^{c}$ Two close-lying states.

* Transitions previously observed in refs. [23-25].

${ }^{131} \mathrm{Cd}$ are listed in table 1 . From data taken in experiments performed at ISOLDE several $\gamma$ rays had already been assigned to the $\beta$ decay of ${ }^{131} \mathrm{Cd}$ prior to the present work: $\gamma$ rays with energies of $844,988,2428,3556,3866,4403$, and $6039 \mathrm{keV}$ in ref. [23], with energies of 995, 1587, 1737, 1910,3560 , and $3870 \mathrm{keV}$ in ref. [24] and finally $\gamma$ rays with energies of $841,2640,3866$, and $6039 \mathrm{keV}$ in ref. [25]. A comparison to table 1 shows that the $988,3556 / 3560$, $3866 / 3870$, and $6039 \mathrm{keV}$ lines most probably correspond to the most intense transitions observed in the present experiment, while the $\gamma$ rays of $841 / 844,995,1587,1737$, $1910,2428,2640$, and $4403 \mathrm{keV}$ are not confirmed by our data.

In the next analysis step coincidence relations were investigated in order to gain additional information with

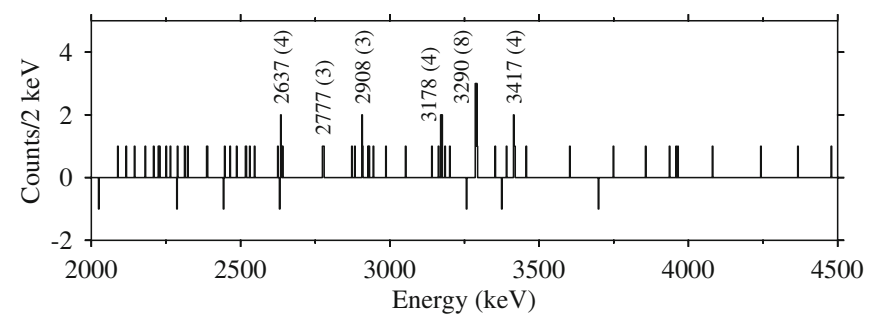

Fig. 3. Spectrum of $\gamma$ rays observed in prompt coincidence with both the first $\beta$ decay observed in WAS3ABi within $300 \mathrm{~ms}$ after the implantation of a ${ }^{131} \mathrm{Cd}$ ion and the detection of the $988 \mathrm{keV}$ transition in the EURICA array. The numbers in brackets behind the energy labels quote the number of observed coincidence events.

respect to the possible placement of the transitions in a level scheme. The $315-3555$ and $433-5525 \mathrm{keV}$ pairs of $\gamma$ rays were found to be in mutual coincidence thus forming cascades in parallel to the observed 3869 (part of the doublet) and $5958 \mathrm{keV}$ transitions. The spectrum of $\gamma$ rays observed in prompt coincidence with the $988 \mathrm{keV}$ $\left(3 / 2^{-}\right) \rightarrow\left(1 / 2^{-}\right)$transition is shown in fig. 3. This spectrum provides weak evidence for coincidence relationships between the $988 \mathrm{keV}$ and the five $\gamma$ rays with energies of $2637,2777,3178,3290$, and $3417 \mathrm{keV}$, which are also seen in fig. 2 and are included in table 1. Furthermore a few counts are found around $2908 \mathrm{keV}$. However, since no clear line is observed at this energy in the $\gamma$-ray spectrum shown in fig. 2 we cannot unequivocally assign this $\gamma$ ray to follow the $\beta$ decay of ${ }^{131} \mathrm{Cd}$. Comparing figs. 2 and 3 we can conclude that none of the intense high-energy transitions above $3.5 \mathrm{MeV}$ decays to the $\left(3 / 2^{-}\right)$state at $1353 \mathrm{keV}$. The $\beta$-decaying ground state of ${ }^{131} \mathrm{Cd}$ is most probably based on a neutron in the $1 f_{7 / 2}$ orbit and therefore has spin $\left(7 / 2^{-}\right)$. Taking into account allowed and first-forbidden decay branches, negative-parity states with spin $5 / 2,7 / 2$, and $9 / 2$ as well as positive-parity levels in the spin range $3 / 2-11 / 2$ are populated in ${ }^{131} \mathrm{In}$. Therefore, both at positive and negative parity, states can be populated which then can decay to both the $\left(9 / 2^{+}\right)$ ground state and the $\left(1 / 2^{-}\right) \beta$-decaying isomer via transitions of relatively low multipolarity. Consequently it is unfortunately not possible to arrange the observed $\gamma$ rays into a decay scheme solely based on the available experimental information. We will come back to this point when we discuss possible configuration assignments in sect. 4.1.

\section{Discussion}

\subsection{Particle-hole configurations in ${ }^{132} \mathrm{Sn}$ and configuration assignments in ${ }^{131}$ In}

In ${ }^{131}$ In core-excited states are expected to lie at excitation energies above $3.5 \mathrm{MeV}$. Indeed, two such states, namely a $\beta$-decaying $21 / 2^{+}$state at $3764 \mathrm{keV}\left(T_{1 / 2}=\right.$ $320(60) \mathrm{ms})$ and a $\left(17 / 2^{+}\right) \gamma$-decaying isomer at $3782 \mathrm{keV}$ $\left(T_{1 / 2}=630(60) \mathrm{ns}\right)$ have been identified in previous works $[6,7]$. It can be assumed that the core-excited states 

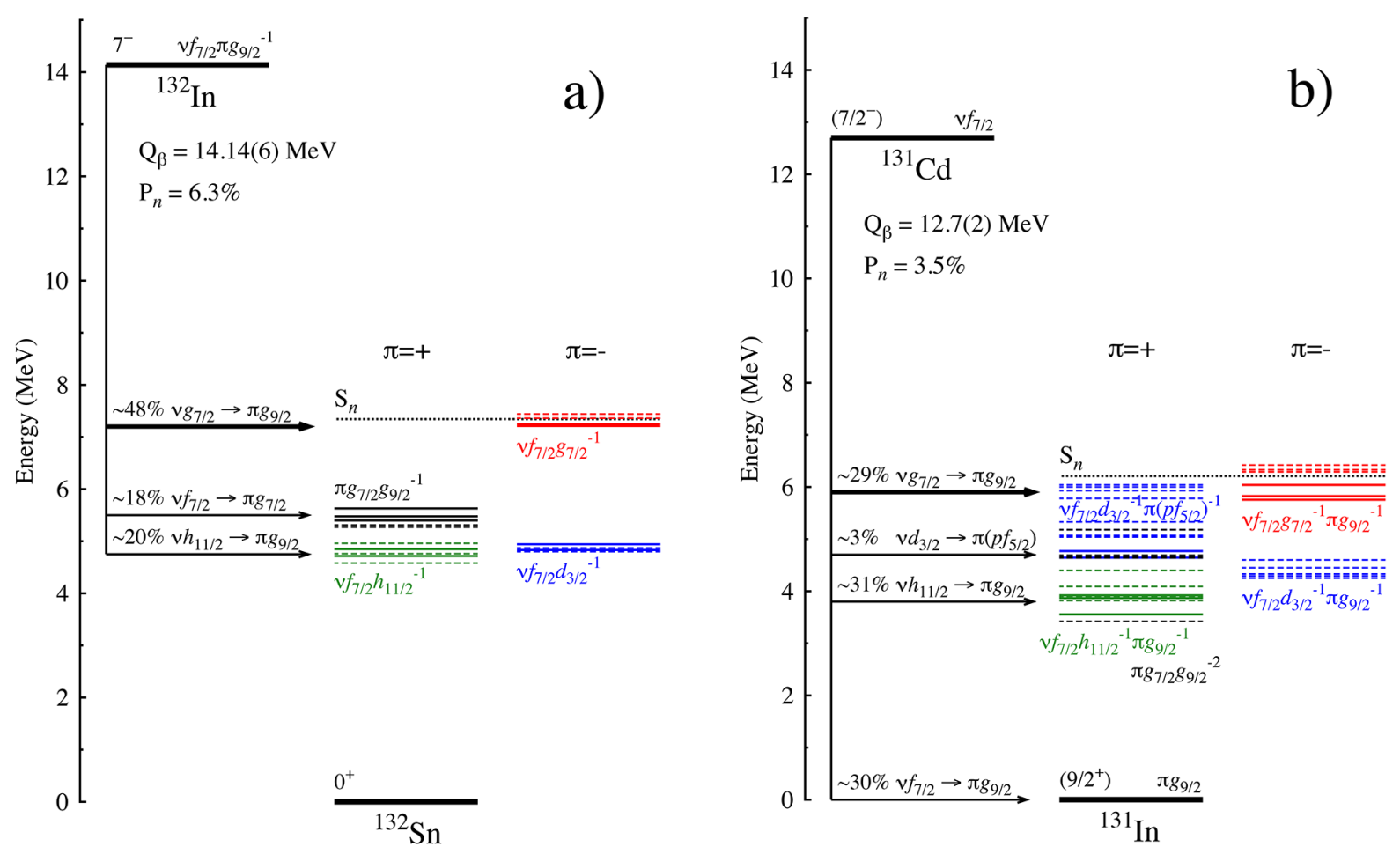

Fig. 4. (Color online) (a) Particle-hole multiplets in the doubly-magic core nucleus ${ }^{132} \mathrm{Sn}$ populated in the $\beta$ decay of ${ }^{132}$ In (adopted from ref. [12]) and (b) schematic excitation scheme of ${ }^{131} \mathrm{In}$ and main decay branches as derived in the present work. The dashed lines show the results obtained for yrast states in shell-model calculations in an extended configuration space (see the text for details), while the dotted lines indicate the neutron separation energies of $S_{n}=7343(7) \mathrm{keV}$ and $S_{n}=6210(40) \mathrm{keV}$ for ${ }^{132} \mathrm{Sn}$ and ${ }^{131} \mathrm{In}$, respectively [26]. The horizontal arrows refer to both columns depending on the configurations. The uppermost arrows (thick lines) in both parts of the figure refer to GT transitions to negative-parity states in the right column, whereas all other arrows (thin lines) refer to ff transitions to positive-parity states in the left column.

consist of a single proton hole in one of the single-particle orbitals below $Z=50$, namely $0 g_{9 / 2}, 1 p_{1 / 2}, 1 p_{3 / 2}$, and $0 f_{5 / 2}$, coupled to the lowest-lying particle-hole multiplets in the ${ }^{132} \mathrm{Sn}$ core nucleus. The structure of excited states in the doubly-magic nucleus ${ }^{132} \mathrm{Sn}$, populated in the $\beta$ decay of ${ }^{132} \mathrm{In}$, has been studied in detail in the past by Björnstad et al. [27] and Fogelberg et al. [12]. In accordance with the magic character of this nucleus, besides the lowest-lying state with negative parity, which was shown to have collective octupole character [12], excited states are found only at energies above $4 \mathrm{MeV}$. On the basis of extensive experimental information, several particle-hole (ph) multiplets were identified. In fig. 4(a) the position of several states belonging to three different neutron- and one proton-ph multiplets in ${ }^{132} \mathrm{Sn}$ is illustrated together with the single-particle transition leading to their population in the $\beta$ decay of ${ }^{132} \mathrm{In}$. The $7^{-}$ground state of ${ }^{132} \operatorname{In}_{83}$ is formed by a proton hole in the $0 g_{9 / 2}$ orbit below $Z=50$ coupled to a neutron in the $1 f_{7 / 2}$ orbit above $N=82$. In about $48 \%$ of all decays of ${ }^{132}$ In two states with spin $6^{-}$and $7^{-}$at excitation energies of 7211 and $7244 \mathrm{keV}$ are populated in ${ }^{132} \mathrm{Sn}$. These states belong to the $\nu\left(f_{7 / 2} g_{7 / 2}^{-1}\right)$ multiplet which is populated via the allowed $\nu 0 g_{7 / 2} \rightarrow \pi 0 g_{9 / 2}$ Gamow-Teller single-particle transition. These $6^{-}, 7^{-}$states decay mainly to the $4^{-}$, $5^{-}$members of the $\nu\left(f_{7 / 2} d_{3 / 2}^{-1}\right)$ multiplet with excitation energies close to $4.9 \mathrm{MeV}$. Note that the latter are not directly populated in the $\beta$ decay of ${ }^{132}$ In. At positive parity, members of two different multiplets are populated via ff transitions. The $6^{+}, 8^{+}$states at around $4.8 \mathrm{MeV}$ belong to the neutron excitation $\nu\left(f_{7 / 2} h_{11 / 2}^{-1}\right)$ populated via the $\nu 0 h_{11 / 2} \rightarrow \pi 0 g_{9 / 2}$ single-particle transition while the $\left(6^{+}, 7^{+}, 8^{+}\right)$states around $5.5 \mathrm{MeV}$ have been assigned in ref. [12] as belonging to the proton-ph multiplet $\pi\left(g_{7 / 2} g_{9 / 2}^{-1}\right)$ populated via the $\nu 1 f_{7 / 2} \rightarrow \pi 0 g_{7 / 2}$ decay.

Turning now to the decay of the $\nu 1 f_{7 / 2}$ ground state of ${ }^{131} \mathrm{Cd}$ we can expect that some of the single-particle transitions observed following the decay of ${ }^{132}$ In will populate three-quasiparticle states at relatively high excitation energies in ${ }^{131} \mathrm{In}$. The strong GT decay $\nu 0 g_{7 / 2} \rightarrow$ $\pi 0 g_{9 / 2}$ would populate members of the $\nu\left(f_{7 / 2} g_{7 / 2}^{-1}\right) \pi g_{9 / 2}^{-1}$ multiplet, which most naturally would decay to the $\left(9 / 2^{+}\right)$ground state. Due to their high intensity we identify the $\gamma$ rays in the $5.5-6.1 \mathrm{MeV}$ range (compare table 1) with these ground state transitions and assign the $\nu\left(f_{7 / 2} g_{7 / 2}^{-1}\right) \pi g_{9 / 2}^{-1}$ configuration to the initial states (see fig. 4(b)). However, we cannot exclude that one of these states is the $\pi 0 g_{7 / 2}$ single-particle state populated via the $\nu 1 f_{7 / 2} \rightarrow \pi 0 g_{7 / 2}$ ff decay branch. This state is expected to lie roughly $6.1 \mathrm{MeV}$ above the $\pi 0 g_{9 / 2}$ ground state [28]. The second ff transition observed in the ${ }^{132} \mathrm{In} \rightarrow{ }^{132} \mathrm{Sn}$ 
Table 2. $\beta$-feeding intensities and $\log f t$ values for the transitions to excited states in ${ }^{131}$ In observed in the $\beta$ decay of ${ }^{131} \mathrm{Cd}$.

\begin{tabular}{ccc|ccc}
\hline$E_{x}(\mathrm{keV})$ & $I_{\beta}(\%)$ & $\log f t$ & $E_{x}(\mathrm{keV})$ & $I_{\beta}(\%)$ & $\log f t$ \\
\hline 0 & $\sim 30$ & $\sim 5.6$ & $4531(8)$ & $1.3(4)$ & $6.1(2)$ \\
$365(8)$ & - & - & $4644(8)$ & $2.3(7)$ & $5.8(2)$ \\
$1353(8)$ & $2.9(20)$ & $6.4(3)$ & $4770(8)$ & $0.7(4)$ & $6.3(3)$ \\
$3480(1)$ & $2.9(7)$ & $6.0(1)$ & $5525(1)$ & $<1.3$ & $>5.9$ \\
$3555(1)$ & $6.4(18)$ & $5.6(1)$ & $5755(1)$ & $5.6(16)$ & $5.2(1)$ \\
$3869(3)^{a}$ & $12.3(19)$ & $5.3(1)$ & $5796(1)$ & $2.5(8)$ & $5.5(2)$ \\
$3921(1)$ & $4.1(10)$ & $5.8(1)$ & $5825(1)$ & $7.7(21)$ & $5.0(1)$ \\
$3990(8)$ & $0.6(2)$ & $6.6(2)$ & $5958(1)$ & $4.6(9)$ & $5.2(1)$ \\
$4038(1)$ & $3.2(13)$ & $5.8(2)$ & $6003(1)$ & $2.9(12)$ & $5.4(2)$ \\
$4130(8)$ & $1.0(5)$ & $6.3(2)$ & $6039(1)$ & $5.5(18)$ & $5.1(2)$ \\
\hline
\end{tabular}

${ }^{a}$ Two close-lying states.

decay, namely $\nu 0 h_{11 / 2} \rightarrow \pi 0 g_{9 / 2}$, populates members of the $\nu\left(f_{7 / 2} h_{11 / 2}^{-1}\right) \pi g_{9 / 2}^{-1}$ multiplet which again, due to the involvement of the $0 g_{9 / 2}$ proton, would preferentially decay to the ground state. We assign the group of strong $\gamma$ rays in the energy range $3.5-4.1 \mathrm{MeV}$ to this decay mode. This scenario is corroborated by comparison to the energy difference of the centroids of the two groups of $\gamma$ rays. From the intensities in table 1 this is calculated as $2.08 \mathrm{MeV}$, which equals the excitation energy of the $\left(\pi g_{9 / 2}^{-1} \nu g_{7 / 2}^{-1}\right) ; I^{\pi}=1^{+}$state relative to the $\left(\pi g_{9 / 2}^{-1} \nu h_{11 / 2}^{-1}\right)$; $I^{\pi}=1^{-}$ground state in ${ }^{130} \mathrm{In}(2.12 \mathrm{MeV})[10]$. The deviation from the $\nu g_{7 / 2}^{-1}-\nu h_{11 / 2}^{-1}$ single-hole energy difference in ${ }^{131} \mathrm{Sn}$ of $2.37 \mathrm{MeV}$ [2] is due to the stronger $\nu g_{7 / 2}-\pi g_{9 / 2}$ $\Delta(N, l)=0$ interaction as compared to the $\nu h_{11 / 2}-\pi g_{9 / 2}$ $\Delta(N, l)=1$ interaction (with $N$ and $l$ denoting here the harmonic oscillator shell main quantum number and the angular momentum, respectively).

Finally, we come back now to the four $\gamma$ rays observed in prompt coincidence with the $988 \mathrm{keV}\left(3 / 2^{-}\right) \rightarrow\left(1 / 2^{-}\right)$ transition as discussed above. Assuming a direct feeding of the $1353 \mathrm{keV}$ state, the 2637 and $2777 \mathrm{keV} \gamma$ rays would originate from levels with excitation energies of 3990 and $4130 \mathrm{keV}$, close to the group of levels in the energy range 3.5-4.1 MeV discussed above. The two coincident 3290 and $3417 \mathrm{keV}$ transitions, on the other hand, define excited states at energies of 4644 and $4770 \mathrm{keV}$, i.e. more than $500 \mathrm{keV}$ above this group. These two states may belong to the $\nu\left(f_{7 / 2} d_{3 / 2}^{-1}\right) \pi\left(p, f_{5 / 2}\right)^{-1}$ multiplets formed by the coupling of a proton in one of the negative-parity orbitals, $1 p_{1 / 2}, 1 p_{3 / 2}$, or $0 f_{5 / 2}$, to the known $\nu\left(f_{7 / 2} d_{3 / 2}^{-1}\right)$ multiplet in the ${ }^{132} \mathrm{Sn}$ core. In this case the decay proceeds via the ff branches $\nu 1 d_{3 / 2} \rightarrow \pi\left(1 p, 0 f_{5 / 2}\right)$.

Table 2 summarizes the $\beta$-feeding intensities and resulting $\log f t$ values for all states included in the tentative level scheme of ${ }^{131} \mathrm{In}$ which is shown in fig. 5. Unfortunately, a direct determination of the intensity of the ground state (g.s.) to ground state decay branch from the

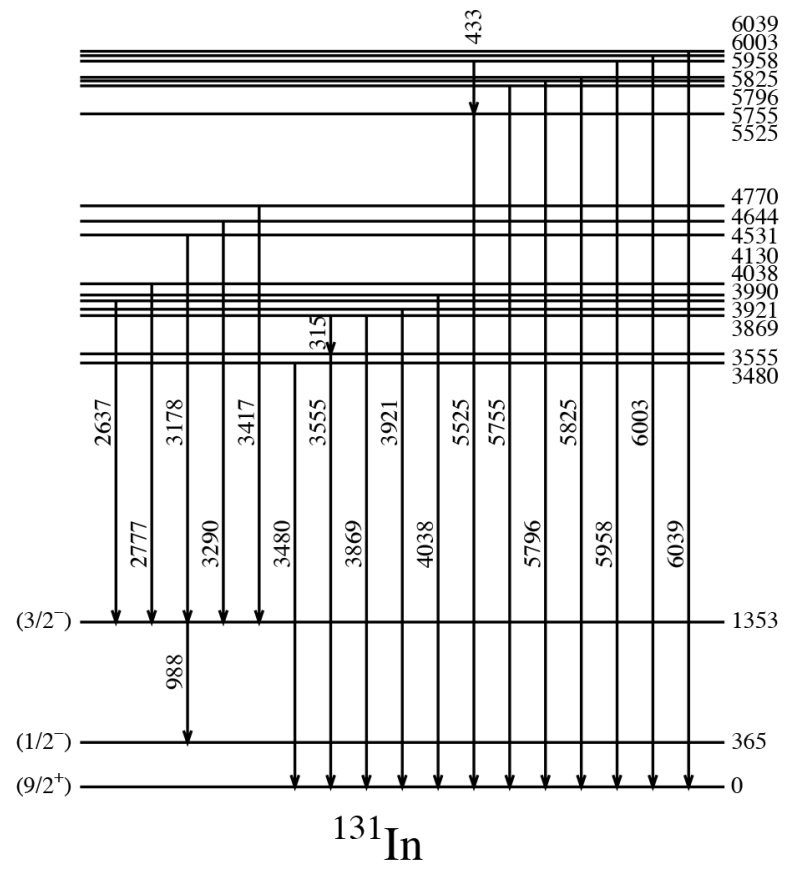

Fig. 5. Tentative excitation scheme of ${ }^{131}$ In established in the present work from the $\beta$ decay of ${ }^{131} \mathrm{Cd}$. The excitation energies of the states, $E_{x}$ in $\mathrm{keV}$, are quoted on the right, the tentative spin assignments on the left-hand side.

data taken in the present experiment is not possible. The $\beta$ feeding to the ground state has therefore been estimated from the sum of the observed feeding to the excited states and the known $\beta$-delayed neutron emission probability of $P_{n}=3.5(10) \%$ [29]. Note that as in all decay studies using high-resolution $\gamma$-ray spectroscopy the log $f t$ values listed in table 2 , and in particular the one for the g.s. to g.s. transition, have strictly speaking to be considered as lower limits due to the Pandemonium effect [30].

\subsection{Comparison to QRPA calculations}

Two different microscopic approaches are commonly used in calculations of $\beta$-decay properties: the shell model (SM) and the quasiparticle random-phase approximation (QRPA). While the former gives more accurate results as compared to QRPA methods, complete shell-model calculations are unfeasible in certain cases, due to computational limitations. In both approaches it has been recognized that the contribution of first-forbidden transitions besides the Gamow-Teller decays is important in some regions of the nuclear chart and the last generations of calculations all include both types of transitions [31-35]. While the most recent shell-model calculations of the $\beta$ decays around ${ }^{132} \mathrm{Sn}$ are limited to $N \leq 82$ nuclei [33], several QRPA calculations crossing $N=82$ are available. QRPA calculations for the GT part with an empirical spreading of the quasiparticle strength are combined with gross theory for the first-forbidden part of the $\beta$ decay in ref. [31]. Borzov et al. calculated the $\beta$-strength functions of the GT and ff decays and the total $\beta$-decay half-lives within the continuum QRPA employing a self-consistent 
approach based on the local energy-density functional theory [32]. QRPA calculations using realistic forces for the proton-neutron interaction have been presented in ref. [34] and finally Niu et al. used a fully self-consistent protonneutron QRPA based on the spherical relativistic HartreeFock-Bogoliubov framework [35].

For ${ }^{131} \mathrm{Cd}$ calculated half-lives were only reported in refs. [31,32]. While $T_{1 / 2}$ values provide information on the average $\beta$ feeding of a nucleus, a comparison between the experimental and theoretical feeding distributions, or the strength functions, allows for a much more stringent test of the nuclear structure input on which the half-life calculations are based on. This has been nicely demonstrated in the work by Hannawald et al. [29], where the half-life, $T_{1 / 2}=68(3) \mathrm{ms}$, and the delayed-neutron emission probability, $P_{n}=3.5(10) \%$, for the $\beta$ decay of ${ }^{131} \mathrm{Cd}$ were experimentally determined and compared with the results of QRPA calculations. In these calculations, several different models were used to calculate the single-particle energies and also the pairing was treated using different approaches. As discussed in detail in ref. [29], the low experimental $P_{n}$ value could only be reproduced by the calculations when a modified Nilsson potential with an $l^{2}$ term reduced by $25 \%$ was used. Only then the lowest threequasiparticle configuration fed by the $\nu 0 g_{7 / 2} \rightarrow \pi 0 g_{9 / 2}$ GT transition (red lines in fig. 4(b)) moved down below the neutron separation energy thus reducing drastically the delayed-neutron emission probability.

In the present work the $\beta$-strength function was calculated within the continuum pnQRPA approach discussed in detail in ref. [32]. This approach has proven to nicely describe the trend of half-lives along the whole chain of Cd isotopes [22]. Values of $Q_{\beta}=13.12 \mathrm{MeV}$ and $S_{n}=5.71 \mathrm{MeV}$ were obtained with the new version of the energy-density functional DF3a, $0.4 \mathrm{MeV}$ higher and $0.5 \mathrm{MeV}$ lower, respectively, as compared to the most recent experimental values of $12.7(2) \mathrm{MeV}$ and $6.210(40) \mathrm{MeV}$ quoted in ref. [26]. The calculated half-life, $T_{1 / 2}=90 \mathrm{~ms}$, and $\beta$-delayed neutron emission probability, $P_{n}=4.6 \%$, nicely reproduce the experimental values of $T_{1 / 2}=98(2) \mathrm{ms}$, obtained from the present data as described in ref. [22], and $P_{n}=3.5(10) \%$ [29]. The theoretical distribution of the $\beta$ strength as a function of the excitation energy in the daughter nucleus as obtained in the continuum QRPA approach is compared to the experimental distribution (calculated from the log $f t$ values quoted in table 2) in fig. 6. This comparison shows that the calculations correctly describe the excitation energies of the three-quasiparticle configurations in ${ }^{131} \mathrm{In}$, though with typically only one excited state per $0.5 \mathrm{MeV}$ bin they do not indicate as much fragmentation as experimentally observed (see fig. 5). Furthermore, the calculations seem to overestimate the absolute strength of the allowed GT transitions in the threshold region and at the same time underestimate the contribution of the first-forbidden decays. Note, however, that this observation may be influenced by the Pandemonium effect [30] mentioned already in the last section. In particular, part of the missing intensity (calculated from the sum of the observed feeding

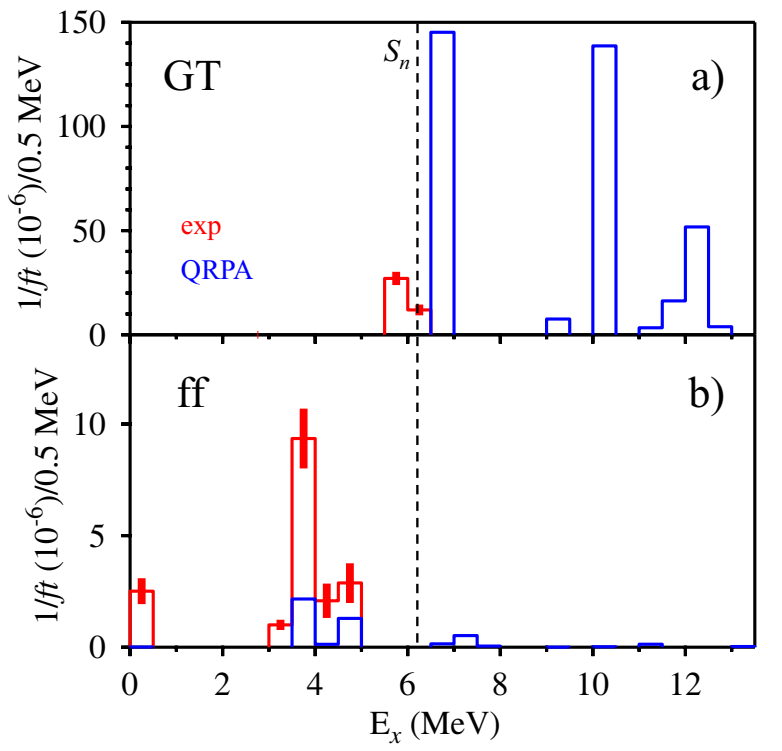

Fig. 6. (Color online) Distribution of the $\beta$ strength for the decay of ${ }^{131} \mathrm{Cd}$ for (a) allowed GT and (b) first-forbidden decay branches. The experimental distributions are shown as red histograms with the vertical bars indicating the error range, while the results of the present QRPA calculations are shown as blue histograms.

to the excited states and the $\beta$-delayed neutron emission probability), which was assigned as ground state feeding in table 2 , could in reality correspond to weak unobserved high-energy $\gamma$ rays feeding the ground state. This open question can be answered only in the future by performing experiments employing the Total Absorption Spectroscopy (TAS) technique [36].

\subsection{Comparison to shell-model calculations}

\subsubsection{Shell-model calculations using realistic interactions}

The description of core-excited configurations such as the ones shown in fig. 4 is not an easy task within the shell model. The configuration space has to be carefully chosen in order to be large enough to include all relevant configurations but still small enough to limit the computational effort. In ref. [7] SM calculations for ${ }^{132} \mathrm{Sn}$ and ${ }^{132} \mathrm{In}$ have been presented which consider the proton $\pi\left(1 p_{1 / 2}, 0 g_{9 / 2}, 0 g_{7 / 2}, 1 d_{5 / 2}\right)$ and neutron $\nu\left(2 s_{1 / 2}, 0 h_{11 / 2}, 1 d_{3 / 2}, 1 f_{7 / 2}, 0 h_{9 / 2}\right)$ model space around ${ }^{132} \mathrm{Sn}$ (see fig. 1), thus allowing for proton excitations across the $Z=50$ as well as neutron excitations across the $N=82$ shell gap, however limiting them to $1 \mathrm{p} 1 \mathrm{~h}$ excitations. In these calculations, which employed twobody matrix elements (TBME) scaled from a realistic interaction for the ${ }^{208} \mathrm{~Pb}$ region and modified to reproduce the positions of the multiplets known in ${ }^{132} \mathrm{Sn}$ (see fig. 4), it was found that the $\nu\left(f_{7 / 2} h_{11 / 2}^{-1}\right) \pi g_{9 / 2}^{-1}$ multiplet in ${ }^{131} \mathrm{In}$ is the lowest one above the first excited state, with its $17 / 2^{+}$and $21 / 2^{+}$members lying close in energy 
to the two observed isomers at 3764 and $3782 \mathrm{keV}$, respectively $[6,7]$. Unfortunately, the $\nu\left(f_{7 / 2} g_{7 / 2}^{-1}\right) \pi g_{9 / 2}^{-1}$ configuration, which is strongly populated in the $\beta$ decay of ${ }^{131} \mathrm{Cd}$ via the $\nu 0 g_{7 / 2} \rightarrow \pi 0 g_{9 / 2}$ GT decay, is outside the model space considered in ref. [7]. Therefore, in the present work the model space was extended to include the $\pi 1 p_{3 / 2}$ and $\nu\left(1 d_{5 / 2}, 0 g_{7 / 2}, 2 p_{3 / 2}\right)$ orbitals. For the latter non-diagonal TBME were limited to $\pi \pi$-hh (hole-hole), $\pi \pi$-pp (particleparticle) and $\nu \nu$-pp configurations, which are needed to correctly treat the pairing. The neglect of the ph mixing by non-diagonal TBME will have a marginal effect on the configuration centroids and the anyway wide spreading. The TBME were obtained from a full set of TBME for the ${ }^{208} \mathrm{~Pb}$ region [37] following the procedure described in ref. [7]. The TBME are extrapolated/interpolated from a full set of TBME for ${ }^{208} \mathrm{~Pb}$ comprising the Kuo-Herling interaction [38] for the pp (north-east) and hh (south-west) space and H7B [39] as described in ref. [37] for the rest (north-west, south-east and cross shell). This combined interaction is part of the OXBASH package as PBALL [40]. For interpolation/extrapolation a method first proposed by Blomqvist [41] was employed. In this method TBME involving $(n, l, j)$ orbits for ${ }^{132} \mathrm{Sn}$ are extrapolated from those with $(n, l+1, j+1)$ for ${ }^{208} \mathrm{~Pb} 1 \hbar \omega$ higher and scaled with $A^{-1 / 3}$ and overlap angle $\theta$ by $\cos \theta$ as described in refs. [7, 42]. Again, the excitation of at most one proton and one neutron across the major shell closures was considered. The results of the calculations for ${ }^{132} \mathrm{Sn}$ and ${ }^{131}$ In $\beta$-decay daughter states in the extended configuration space, which have been performed with the computer code OXBASH [40], are included as dashed lines in fig. 4. For ${ }^{131}$ In only yrast states are shown. As already observed for the core-excited states studied in ref. [7], the SM calculations tend to overestimate the experimental excitation energies in ${ }^{131} \mathrm{In}$. This deficiency can be attributed to the restriction to $t=1$ truncation as discussed in detail for the case of the core-excited $12^{+}$spin-gap isomer in ${ }^{98} \mathrm{Cd}$ in ref. [43]. This overestimation is especially delicate in the region close to the neutron separation energy and prohibits a reliable estimation of the $\beta$-delayed neutron emission probability on the basis of the present SM calculations. We note in passing that any minor uncertainty in the effective interaction employed introduces a similar sensitivity of predicted $\beta$ n ratios without invoking shell gap quenching [29].

A closer inspection of fig. 4(b) shows that the calculated states of the $\nu\left(f_{7 / 2} d_{3 / 2}^{-1}\right) \pi p^{-1}$ multiplet (blue dashed lines) fall into two groups, corresponding to states involving a proton hole in either the $\pi 1 p_{1 / 2}$ or the $\pi 1 p_{3 / 2}$ orbit. From the position of these states it seems likely that the $\nu\left(f_{7 / 2} d_{3 / 2}^{-1}\right) \pi f_{5 / 2}^{-1}$ configuration, populated in the $\nu 1 d_{3 / 2} \rightarrow \pi 0 f_{5 / 2}$ decay, is shifted beyond the neutron separation energy and therefore does not have to be considered. Besides the configurations already discussed in sect. 4.1 also the calculated $3 / 2^{+}-11 / 2^{+}$members of the $\pi g_{7 / 2} g_{9 / 2}^{-2}$ proton multiplet in ${ }^{131} \mathrm{In}$, which are the daughter states of the $\nu 1 f_{7 / 2} \rightarrow \pi 0 g_{7 / 2}$ ff decay, are included in fig. 4(b) (black dashed lines). Except for the $3 / 2^{+}$ state, these will decay by $M 1$ and/or $E 2$ transitions to the $\pi 0 g_{9 / 2}^{-1}$ ground state. Due to the wide energy spread within all three calculated core-excited configurations at positive parity and the resulting overlap, the configuration assignments to the experimentally identified states discussed in sect. 4.1 clearly have to be considered tentative.

At negative parity, the states belonging to the $\nu\left(f_{7 / 2} d_{3 / 2}^{-1}\right) \pi g_{9 / 2}^{-1}$ multiplet are not populated directly in the $\beta$ decay, but could in principle be populated via $\nu 0 g_{7 / 2} \rightarrow \nu 1 d_{3 / 2} \quad E 2$ transitions in the $\gamma$ decay of the $\nu\left(f_{7 / 2} g_{7 / 2}^{-1}\right) \pi g_{9 / 2}^{-1}$ states, in competition to the high-energy $\nu 1 f_{7 / 2} \rightarrow \nu 0 g_{7 / 2}$ decay to the ground state. Note that in the decay of the $\nu f_{7 / 2} g_{7 / 2}^{-1}$ multiplet in ${ }^{132} \mathrm{Sn}$ the $\nu 0 g_{7 / 2} \rightarrow$ $\nu 1 d_{3 / 2}$ decay branch is indeed observed [12]. The $2005 \mathrm{keV}$ $\gamma$ ray, which is included in table 1 but not arranged in the level scheme of fig. 5, as well as additional weaker transitions visible in fig. 2 in the energy range $1.7-2.3 \mathrm{MeV}$ could correspond to decays of the $\nu\left(f_{7 / 2} g_{7 / 2}^{-1}\right) \pi g_{9 / 2}^{-1}$ multiplet to either the $\nu\left(f_{7 / 2} d_{3 / 2}^{-1}\right) \pi g_{9 / 2}^{-1}$ or $\nu\left(f_{7 / 2} h_{11 / 2}^{-1}\right) \pi g_{9 / 2}^{-1}$ states.

\subsubsection{Shell-model calculations using schematic interactions}

An alternative shell-model approach to the study of ${ }^{131}$ In has recently been presented by Wang et al. [44]. In this work, a large model space consisting of the $\pi\left(0 f_{5 / 2}, 1 p_{3 / 2}, 1 p_{1 / 2}, 0 g_{9 / 2}, 0 g_{7 / 2}, 1 d_{5 / 2}\right)$ and $\nu\left(0 g_{7 / 2}\right.$, $\left.1 d_{5 / 2}, 2 s_{1 / 2}, 0 h_{11 / 2}, 1 d_{3 / 2}, 1 f_{7 / 2}, 2 p_{3 / 2}\right)$ single-particle orbitals outside a ${ }^{78} \mathrm{Ni}$ core is considered, which allows for a consistent description of both low-lying and highenergy states in nuclei below ${ }^{132} \mathrm{Sn}$. As the effective interaction, an extended pairing-plus-quadrupole force is employed, with monopole corrections introduced to adjust the positions of the $21 / 2^{+}$and $17 / 2^{+}$states of the $\nu\left(f_{7 / 2} h_{11 / 2}^{-1}\right) \pi g_{9 / 2}^{-1}$ configuration to the experimental values. In fact, these two states were originally calculated around $4.5 \mathrm{MeV}$, i.e. about $0.7 \mathrm{MeV}$ above the observed excitation energies. With the size of the $N=82$ shell gap fixed through the monopole correction terms in the Hamiltonian, the isomerism along the $N=82$ isotonic chain [45] and the quenching of the $N=82$ shell gap near ${ }^{120} \mathrm{Sr}[46]$ have been discussed.

The lowest-lying negative-parity states of the $\nu\left(f_{7 / 2} h_{11 / 2}^{-1}\right) \pi p_{1 / 2}^{-1}$ configuration are found to lie around $4.3 \mathrm{MeV}$. This is at variance with the results of ref. [7], where these states were predicted at $\sim 0.4-0.7 \mathrm{MeV}$ above the negative-parity ones arising from the $\nu\left(f_{7 / 2} d_{3 / 2}^{-1}\right) \pi g_{9 / 2}^{-1}$ configuration, which was found to be located at $\sim 4.4 \mathrm{MeV}$ excitation energy. As for the $5 / 2^{-}, 7 / 2^{-}, 9 / 2^{-}$states of the $\nu\left(f_{7 / 2} g_{7 / 2}^{-1}\right) \pi g_{9 / 2}^{-1}$ configuration, corresponding to the observed levels just below the neutron separation energy of ${ }^{131} \mathrm{In}$ (see fig. 4), the schematic interaction used in ref. [44] predicts excitation energies above $7 \mathrm{MeV}$ [47], which is more than $1 \mathrm{MeV}$ above the experimental values.

The above discussion seems to evidence the necessity to introduce additional, configuration-dependent 
monopole corrections besides the ones applied in [44]. Actually, within this SM approach one should adjust the schematic interaction to as much as possible experimental information before the predictions of such calculations for experimentally unaccessible regions of the chart of nuclei can be relied upon.

\subsection{Non-observation of the $0 \mathrm{f}_{5 / 2}$ proton-hole state}

As stated in the beginning of the article, one of the goals of the present work was to identify the $1 p_{3 / 2}$ and $0 f_{5 / 2}$ proton-hole states with respect to the ${ }^{132} \mathrm{Sn}$ core. While the $\left(3 / 2^{-}\right)$state was observed at an excitation energy of $1353 \mathrm{keV}$ [13], the 5/2- level remains unobserved. At first sight one may expect the latter to be directly populated in the $\beta$ decay of ${ }^{131} \mathrm{Cd}$ via a $\nu 1 f_{7 / 2} \rightarrow \pi 0 f_{5 / 2}$ GT transition. However, two facts prevent such a direct population: first, the $\pi 0 f_{5 / 2}$ orbital is almost filled and second, the two orbitals differ by two harmonic oscillator shells with a vanishing single-particle transition due to the orthogonality of the radial wave functions. The presence of two orbitals close to the Fermi surface, despite protons and neutrons, that differ by $2 \hbar \omega$ is somewhat unique in the Segré chart that occurs only once more, namely in the $\mathrm{Pb}$ region with the $\nu 1 g_{9 / 2}$ and $\pi 0 g_{7 / 2}$ orbitals. And indeed, in the ${ }^{207} \mathrm{Hg} \rightarrow{ }^{207} \mathrm{Tl}$ decay, which is the analog to the ${ }^{131} \mathrm{Cd} \rightarrow{ }^{131} \mathrm{In}$ decay in the ${ }^{208} \mathrm{~Pb}$ region, the known $\pi 0 g_{7 / 2}$ state at an excitation energy of $3.47 \mathrm{MeV}$ is not populated [48].

On the other hand, the SM calculations discussed in sect. 4.3.1 clearly show that there are plenty of states populated in the $\beta$ decay of ${ }^{131} \mathrm{Cd}$ which, via mixing, could feed a $5 / 2^{-}$state with main configuration $\pi 0 f_{5 / 2}^{-1}$. Even in this truncated approach 101 daughter states of $\mathrm{ff}$ decays in the spin range $3 / 2^{+}-11 / 2^{+}$(57 for spins $3 / 2^{+}-7 / 2^{+}$) and 52 GT daughter states with spins of $5 / 2^{-}, 7 / 2^{-}$, or $9 / 2^{-}$are obtained. To assess the decay pattern of the $5 / 2^{-}$ state SM calculations in the pure $N=82$ proton hole space $\pi\left(0 f_{5 / 2}, 1 p_{3 / 2}, 1 p_{1 / 2}, 0 g_{9 / 2}\right)$ were performed with the NA14 interaction introduced in refs. [11, 13]. Standard values of the effective charge, $e_{\pi}=1.5 e$, and $g$ factor, $g_{s}=0.7 g_{s}^{\text {free }}$, were assumed. The calculations show that the $E 2$ decay branch from the $5 / 2^{-}$to the $1 / 2^{-}$state at an excitation energy of $365 \mathrm{keV}$ dominates (90-95\%), while the $M 2$ branch to the $9 / 2^{+}$ground state is expected equal to or even stronger than the $l$-forbidden $M 1$ to the $3 / 2^{-}$level at $1353 \mathrm{keV}$, which is reduced to its $E 2$ component (both $\leq 5 \%$ ). Since the main decay branch populates a $\beta$-decaying state, the $\gamma$ emission from the $5 / 2^{-}$protonhole state is not observed in coincidence with any other intense $\gamma$ ray. The only signature of such a state would be the observation of two $\gamma$ rays differing in energy by $365 \mathrm{keV}$ (and possibly a third one with an energy smaller by 988 respectively $1353 \mathrm{keV}$ ). Therefore, in order to fix the position of the last missing proton-hole state with respect to the ${ }^{132} \mathrm{Sn}$ core, i.e. the energy of the $\pi 0 f_{5 / 2}$ orbital, in the $\beta$ decay of ${ }^{131} \mathrm{Cd}$, an experiment with much higher statistics as compared to the one presented here will be necessary.

\section{Conclusions}

We reported on a study of the $\beta$ decay of ${ }^{131} \mathrm{Cd}$. Three single-particle transitions are found to strongly contribute to this decay, namely the allowed $\nu 0 g_{7 / 2} \rightarrow \pi 0 g_{9 / 2}$ GT decay and the two first-forbidden transitions $\nu 0 h_{11 / 2} \rightarrow$ $\pi 0 g_{9 / 2}$ and $\nu 1 f_{7 / 2} \rightarrow \pi 0 g_{9 / 2}$. Besides the $1 p_{3 / 2}$ protonhole state already reported in ref. [13], we identified core-excited states belonging to the $\nu\left(f_{7 / 2} h_{11 / 2}^{-1}\right) \pi g_{9 / 2}^{-1}$, $\nu\left(f_{7 / 2} d_{3 / 2}^{-1}\right) \pi\left(p, f_{5 / 2}^{-1}\right)$ and $\nu\left(f_{7 / 2} g_{7 / 2}^{-1}\right) \pi g_{9 / 2}^{-1}$ configurations, for the first time in the case of the two last configurations. Their excitation energies were compared to predictions of QRPA and shell-model calculations and a good overall agreement was found. Realistic SM calculations in an extended configuration space considering the excitation of at most one proton and one neutron across the $Z=50$ and $N=82$ shell closures slightly overestimate the experimental excitation energies in ${ }^{131} \mathrm{In}$. On the other hand, it was shown that in order to describe core-excited states in this nucleus employing schematic forces, it is essential to apply configuration-dependent corrections.

We thank the staff of the RIKEN Nishina Center accelerator complex for providing stable beams with high intensities for the experiment. We acknowledge the EUROBALL Owners Committee for the loan of germanium detectors and the PreSpec Collaboration for the readout electronics of the cluster detectors. This work was supported by the Spanish Ministerio de Ciencia e Innovación under contract FPA2011-29854-C04 and the Spanish Ministerio de Economía y Competitividad under contract FPA2014-57196-C5-4-P, the Generalitat Valenciana (Spain) under grant PROMETEO/2010/101, the National Research Foundation of Korea (NRF) grant funded by the Korea government (MEST) (No. NRF-2014S1A2A2028636), the Priority Centers Research Program in Korea (2009-0093817), OTKA contract number K-100835, JSPS KAKENHI (Grant No. 25247045), the Grant by IN2P3-RFBR under Agreement No. 110291054, the STFC (UK), the European Commission through the Marie Curie Actions call FP7-PEOPLE-2011IEF under Contract No. 300096, the U.S. Department of Energy, Office of Nuclear Physics, under Contract No. DE-AC0206CH11357, the "RIKEN foreign research program" and the German BMBF (No. 05P12RDCIA and 05P12RDNUP) and HIC for FAIR.

\section{References}

1. K.L. Jones et al., Nature 465, 454 (2010).

2. B. Fogelberg et al., Phys. Rev. C 70, 034312 (2004).

3. M. Sanchez-Vega et al., Phys. Rev. C 60, 024303 (1999).

4. B. Fogelberg, Y. Zongyuan, L. Spanier, Phys. Lett. B 209, 173 (1988).

5. A. Kankainen et al., Phys. Rev. C 87, 024307 (2013).

6. B. Fogelberg et al., Nucl. Phys. A 429, 205 (1984).

7. M. Górska et al., Phys. Lett. B 672, 313 (2009).

8. H. Gausemel et al., Phys. Rev. C 69, 054307 (2004).

9. B. Fogelberg, in Proceedings of the International Conference on Nuclear Data for Science and Technology, Mito, Japan, 1988 (Japan Atomic Energy Research Institute) p. 873 . 
10. I. Dillmann et al., Phys. Rev. Lett. 91, 162503 (2003).

11. J. Taprogge et al., Phys. Rev. C 91, 054324 (2015).

12. B. Fogelberg et al., Phys. Rev. Lett. 73, 2413 (1994).

13. J. Taprogge et al., Phys. Rev. Lett. 112, 132501 (2014).

14. S. Nishimura et al., Prog. Theor. Exp. Phys. 2012, $03 \mathrm{C} 006$ (2012).

15. S. Nishimura et al., RIKEN Accel. Prog. Rep. 46, 182 (2013).

16. P.-A. Söderström et al., Nucl. Instrum. Methods B 317, 649 (2013) (XVIth International Conference on ElectroMagnetic Isotope Separators and Techniques Related to their Applications, December 2-7, 2012 at Matsue, Japan).

17. T. Kubo et al., Prog. Theor. Exp. Phys. 2012, $03 \mathrm{C} 003$ (2012).

18. N. Fukuda et al., Nucl. Instrum. Methods B 317, 323 (2013) (XVIth International Conference on ElectroMagnetic Isotope Separators and Techniques Related to their Applications, December 2-7, 2012 at Matsue, Japan).

19. A. Jungclaus et al., Phys. Rev. C 93, 041301(R) (2016).

20. J. Eberth et al., Nucl. Instrum. Methods Phys. Res. A 369, 135 (1996).

21. J. Simpson, Z. Phys. A 358, 139 (1997).

22. G. Lorusso et al., Phys. Rev. Lett. 114, 192501 (2015).

23. O. Arndt et al., Acta Phys. Pol. B 40, 437 (2009).

24. Michael Walter Hannawald, $\mathrm{PhD}$ Thesis, Johannes Gutenberg-Universität Mainz (2000) pp. 127-128.

25. Carola Jost, PhD Thesis, Johannes Gutenberg-Universität Mainz (2010) pp. 103-108.

26. M. Wang et al., Chin. Phys. C 36, 1603 (2012).

27. T. Björnstad et al., Nucl. Phys. A 453, 463 (1986).

28. H. Grawe, K. Langanke, G. Martínez-Pinedo, Rep. Prog. Phys. 70, 1525 (2007).
29. M. Hannawald et al., Phys. Rev. C 62, 054301 (2000).

30. J. Hardy, L. Carraz, B. Jonson, P. Hansen, Phys. Lett. B 71, 307 (1977).

31. P. Möller, B. Pfeiffer, K.-L. Kratz, Phys. Rev. C 67, 055802 (2003).

32. I.N. Borzov et al., Nucl. Phys. A 814, 159 (2008).

33. Q. Zhi et al., Phys. Rev. C 87, 025803 (2013).

34. D.-L. Fang, B.A. Brown, T. Suzuki, Phys. Rev. C 88, 034304 (2013).

35. Z.M. Niu et al., Phys. Lett. B 723, 172 (2013).

36. C.L. Duke et al., Nucl. Phys. A 151, 609 (1970).

37. E.K. Warburton, Phys. Rev. C 44, 233 (1991).

38. T.T.S. Kuo, G.H. Herling, U.S. Naval Research Laboratory Report No. 2258 (1971).

39. A. Hosaka, K.-I. Kubo, H. Toki, Nucl. Phys. A 76, 444 (1985).

40. B.A. Brown et al., MSU-NSCL Report No. 1289 (2009).

41. J. Blomqvist, CERN Report No. 81-09 (CERN, Geneva, 1981) p. 535.

42. H. Grawe, Lect. Notes Phys. 651, 33 (2004).

43. A. Blazhev et al., Phys. Rev. C 69, 064304 (2004).

44. H.-K. Wang et al., Phys. Rev. C 88, 054310 (2013).

45. H.-K. Wang, K. Kaneko, Y. Sun, Phys. Rev. C 89, 064311 (2014).

46. H.-K. Wang, K. Kaneko, Y. Sun, Phys. Rev. C 91, 021303(R) (2015).

47. Yang Sun, private communication.

48. B. Jonson, O.B. Nielsen, L. Westgaard J. Zylicz, in Proceedings of the International Conference on Nuclei Far from Stability, Helsingor, Denmark, Vol. 2 (CERN, Service d'information scientifique, 1981) p. 640. 